\title{
Abdominoplasty in Prune Belly Syndrome: Umbilicus Reconstruction Using Modified Montfort Technique
}

\author{
Luiz G Freitas Filho*, Karine Furtado Meyer, Mila Torii Correa Leite and Luiz J Budib \\ Department of Urology - Hospital Santa Marcelina, Universidade Federal de São Paulo, Brazil
}

Submission: July 13, 2018; Published: September 19, 2018

*Corresponding author: Luiz G Freitas Filho, Department of Urology - Hospital Santa Marcelina, Brazil, Tel: 5511 983231299;

Email: luizfreitasepm@gmail.com.br

Abstract

Introduction: Abdominoplasty is a major component in the management of the Prune Belly syndrome (PBS). Traditional techniques reinforce the muscular wall, however maintaining the original umbilicus positioned more cranially than usually and most frequently presenting a bizarre aspect.

Case report: We report on the case of a PBS patient, who underwent correction of the abdominal wall under a technical variant of Monfortâ $€^{\mathrm{TM}} \mathrm{s}$ intervention and whose cosmetic aspect, twenty-three years following the surgery, is considered outstanding.

Discussion: The Prune Belly Syndrome is a rare condition that involves different organs and whose esthetic aspect affects the body image. We propose a modification to the technique based on full reconstruction of the umbilical scar by using either circular or semicircular flaps from the lateral skin of the abdomen.

Keywords: Prune belly syndrome; Abdomen; Surgery; Umbilicus

\section{Introduction}

The Prune Belly syndrome (PBS) involves a broad aspect of alterations, the most important of which being abdominal musculature deficiency, bilateral testicular dystopia and an abnormal urinary tract. A case, described by Osler [1], addressing the characteristic findings of the disease made the term "Prune Belly Syndrome" popular.

We report on the case of a PBS patient, who underwent correction of the abdominal wall under a technical variant of Monforte's intervention and whose cosmetic aspect, twentythree years following the surgery, is considered good.

\section{Case Report}

Male patient, born on the 10th of October 1994 with PBS. At birth his urine was noted to drain through the urachus; he had urethral atresia. At one week of life he underwent vesicostomy and at one month he had an only preputial flap applied to the urethra previously opened on its ventral fascia (Figure1(A-C)). At three months of life the vesicostomy was closed after resection of the bladder dome. At 1 year he had bilateral urethroplasty with ureter vesical reimplantation by Cohen's technique, bilateral orchidopexy and abdominoplasty by modified Montfort technique (Figure 2(A-C)). In early childhood he presented some episodes of urinary tract infections, due to a significant urinary residue that was treated with intermittent catheterization. While growing he started urinating adequately and urinary catheterization was discontinued. Currently, twenty-three years after the surgery, he urinates normally, has normal renal function, just presenting ureter hydronephrosis at US examination. The aspect of the abdomen is good, as shown in (Figure 3(A-C)).

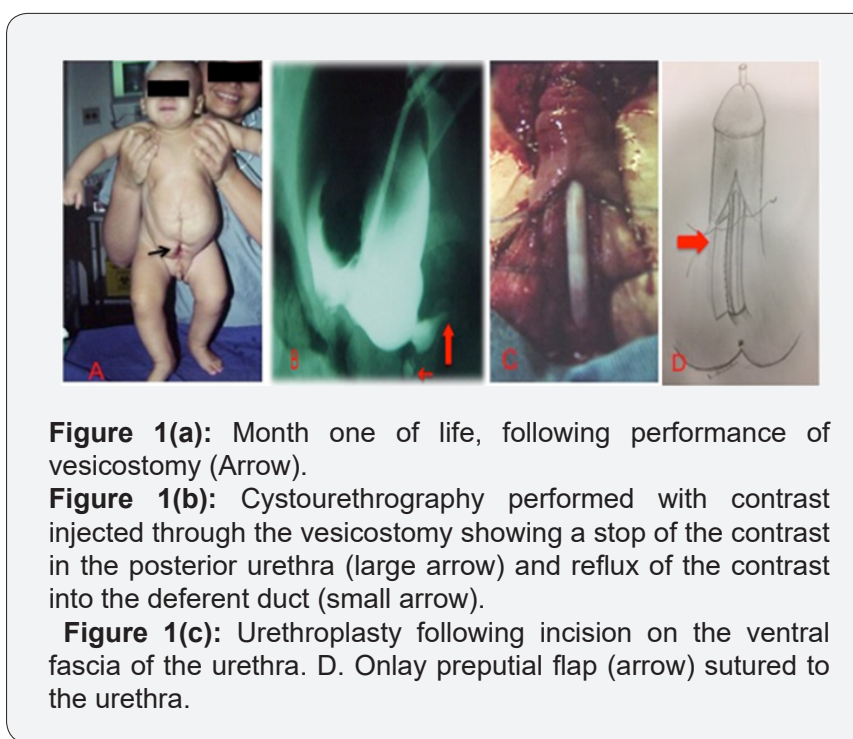

\section{Abdominoplasty}

Instead of maintaining the umbilical scar, always quite above the usual position, the entire skin of the central area of the 
abdomen is excised and the umbilicus is reconstructed by using two circular flaps that are sutured together and then fixed to the reconstructed musculature, as shown in (Figure 2(A-B)). Two lateral muscular incisions are then performed under Montfort technique.

\section{Discussion}
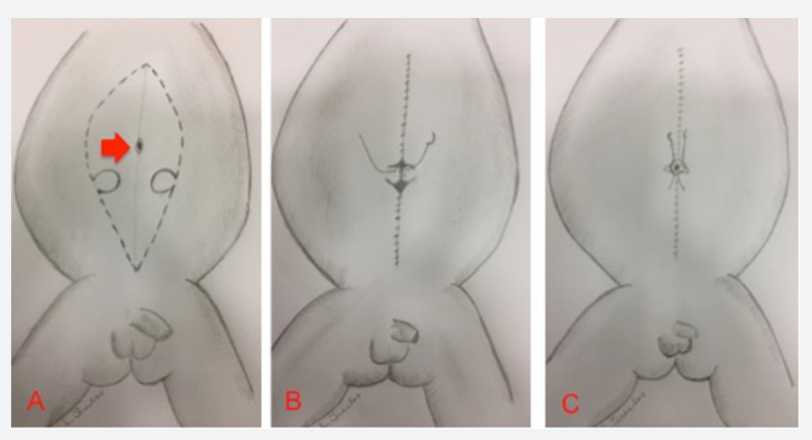

Figure 2(a): Incision line drawn with both flaps at the new umbilical scar position. Note the more cranial position of the original umbilicus (Arrow).

Figure 2(b): Suture of both circular flaps and fixation to the musculature.

Figure 2(C): Confection of a continuous suture for final creation of the new umbilicus.
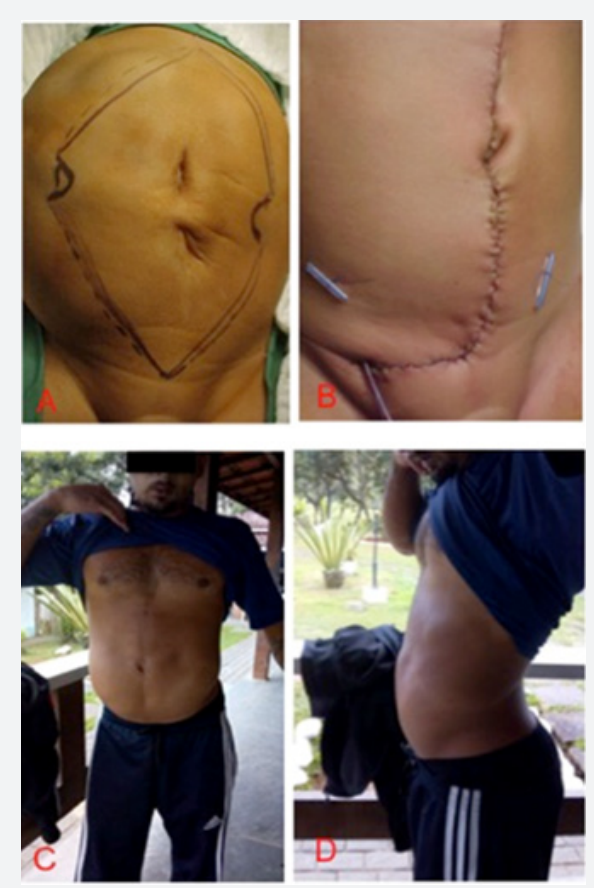

Figure 3(a): Marking of the abdominal incision with the circular flaps. Figure 3(b): Immediate postoperative result.

Figure 3(C\& D): Result 23 years after the abdominoplasty.

The Prune Belly Syndrome is a rare condition that involves different organs and whose esthetic aspect affects the body image and quality of life of patients and of their caregivers. Abdominal wall laxity is a major component of the syndrome and the more cranial position of the umbilical scar, usually of a bizarre aspect, has not been corrected in traditional techniques [2-4]. Randolff et al. [3] drew an electromyographic mapping of the PBS patients and showed the medial and lower muscle segments to be the more consistently affected ones. The technique described by Monforte [2] increases and strengthens the muscle wall thickness, however preserving the umbilical scar in its original position, usually quite above the iliac crests line. We propose a modification to the technique based on full reconstruction of the umbilical scar by using either circular or semicircular flaps from the lateral skin of the abdomen (Figure 2 \& 3). The initial result led us to use the technique in other PBS patients and more recently in patients with bladder exstrophy whose umbilical scar is lower, with quite encouraging results. 


\section{Ethical Approval}

All procedures performed in the study were in accordance with the ethical standards of the institutional and national research committee and with the 1964 Helsinki declaration and its later amendments on comparable ethical standards.

\section{References}

1. Osler WO Congenital absence of abdominal muscles, with distended and hypertrophied urinary bladder. Bull Johns Hopkins Hosp 1901(12): 331-333.
2. Monfort G, Guys JM, Bocciardi A, Coquet M, Chevalier D (1991) A novel technique for the reconstruction of the abdominal wall in the prune belly syndrome. J Urol 146(2 Pt 2): 639-640.

3. Randolf J, Cavett C, Eng G (1981) Abdominal wall reconstruction in the prune belly syndrome. J Pediatr Surg 16(6): 960-964.

4. Smith EA, Srinivasan A, Schrerz HC, Tracey AJ, Broecker B, et al. (2017) Abdominoplasty in prune belly syndrome: modifications in Monfort technique to address variable patterns of abdominal wall weakness. J Pediatr Urol 13(5): 502e1-502e6.

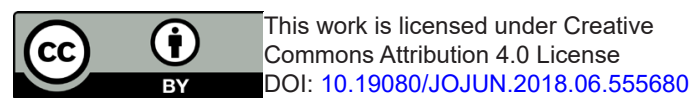

